\title{
Expanding the reach of the p53 tumor suppressor network
}

\author{
GP Zambetti ${ }^{*, 1}$ \\ Cell Death and Differentiation (2014) 21, 505-506. doi:10.1038/cdd.2014.13
}

Lying at the heart of most human cancers is the p53 tumor suppressor pathway. ${ }^{1}$ When functional, p53 responds to a wide array of cellular stresses by inducing cell cycle arrest or apoptosis. It also facilitates DNA repair, regulates cell metabolism, blocks angiogenesis and triggers cell senescence. Therefore, it is not surprising that when p53 is mutated an emerging tumor cell, perhaps as a result of the activation of an oncogene, can continuously divide, survive and undergo metastasis. How p53 controls such essential processes to suppress tumorigenesis is primarily based on its ability to transcriptionally regulate an ever-expanding series of downstream target genes. The study by Wang et al. ${ }^{2}$ has taken an integrative genomic approach using chromatin immunoprecipitation-coupled sequencing (ChIP-seq) and transcriptome analyses to identify an extensive panel of new target genes that may mediate established p53 activities and less well-characterized p53 functions involved in cell signaling, metabolism, motility and immunity. These findings open new opportunities for advancing our understanding of how the p53 network protects against oncogenesis.

We have learned much since the identification of the p53 DNA-binding consensus element (RRRCWWGYYY) ${ }^{3}$ and the first recognized p53 responsive gene $p 21^{C I P 1}(C D K N 1 A),{ }^{4}$ which plays a major role in enforcing a $\mathrm{G} 1$ cell cycle arrest upon DNA damage. Indeed, there are now approximately 150 genes that have been reportedly regulated by the p53 tumor suppressor. The degree to which each of these genes has been validated as a bona fide target is variable, raising an important question as to their relevancy within the p53 network. In addition to $p 21^{C I P 1}$, some other well-established targets that have been genetically and biochemically validated include Mdm2 (an essential negative regulator of p53) and PUMA (encoding pro-apoptotic BH3-only protein). Each of these genes harbors variations on the theme of the p53 consensus site within their promoter-regulatory regions. Some have higher-affinity sites and are more responsive, whereas others have lower-affinity sites and are less responsive to p53. Therefore, the level of p53 protein could dictate the response, as elegantly shown by Prives and co-workers ${ }^{5}$ using conditional expression cell models. Further influencing the capacity of a target to respond to p53 are the surrounding promoter sequences (NB, some elements are located downstream within introns), the cell type (e.g., fibroblasts versus lymphocytes) and the magnitude and form of cell stress (e.g., DNA damage versus oncogenemediated hyperproliferation).

We have also learned that not all p53 mutations are created equal. Initial studies focused on the hotspot mutants (e.g., $\mathrm{R} 175 \mathrm{H}$ ) that contain a missense mutation in the DNA-binding domain. In general, these mutants are incompetent for binding to DNA and activating downstream target genes. There are now more than 28000 TP53 mutations that have been detected in human cancers (IARC TP53 Database). ${ }^{6}$ Some mutations (e.g., R175P) compromise p53 apoptotic activity, but not its ability to block cell proliferation. ${ }^{7}$ Others, such as the $\mathrm{R} 337 \mathrm{H}$ founder germline mutation in southern Brazil, have little consequence on p53 activity, at least in cell-based assays, but have a profound effect on the susceptibility of childhood adrenocortical carcinoma and other tumor types. ${ }^{8,9}$ These findings establish a hierarchy of p53 mutations based on structure, function and cancer risk. ${ }^{10}$

Ishioka and colleagues ${ }^{11}$ interrogated the transcriptional competency of more than 2300 mutants, including multiple amino-acid substitutions at the same site (e.g., $\mathrm{R} 175 \mathrm{H}$ and $\mathrm{R} 175 \mathrm{P}$ ), in a yeast model system. The results of this study have been compiled at the IARC TP53 Database and serve as a good first check for whether a mutation identified in a tumor is functional or not. The present study by Wang et al. ${ }^{2}$ took a similar, but more physiological approach in mammalian cells. Wild-type p53 (WTp53) and 16 naturally occurring variants resulting from non-synonymous SNPs were evaluated for transactivation of the p53 response element (RE) and all combinations of the CWWG core motif, as well as several established promoter reporters (e.g., p21 cip1, PLK3 and RNF144B). In parallel, WTp53 and the variants were tested for DNA binding to the p53RE panel. The primary approach was to assess the function of exogenous WTp53 and each variant in human colon carcinoma HCT116 $\mathrm{p53}^{-/-}$cells. Eleven of the sixteen variants exhibited WTp53 activities, whereas five displayed a loss of function and were classified as null (all associated with SNPs within the DNA binding domain). The two groups of variants (functional and nonfunctional) in effect represent multiple independent replicates of WTp53 and negative controls for testing in gene expression-profiling studies. Genome-wide ChIP-seq analysis using WTp53 and the variants identified 592 novel p53-responsive

\footnotetext{
${ }^{1}$ Department of Biochemistry, St. Jude Children's Research Hospital, Memphis, TN 38105, USA

${ }^{*}$ Corresponding author: GP Zambetti, Department of Biochemistry, St. Jude Children's Research Hospital, Memphis, TN 38105 , USA. Tel: +1 9015953429 ; Fax: +1 901525 8025; E-mail: gerard.zambetti@stjude.org
} 


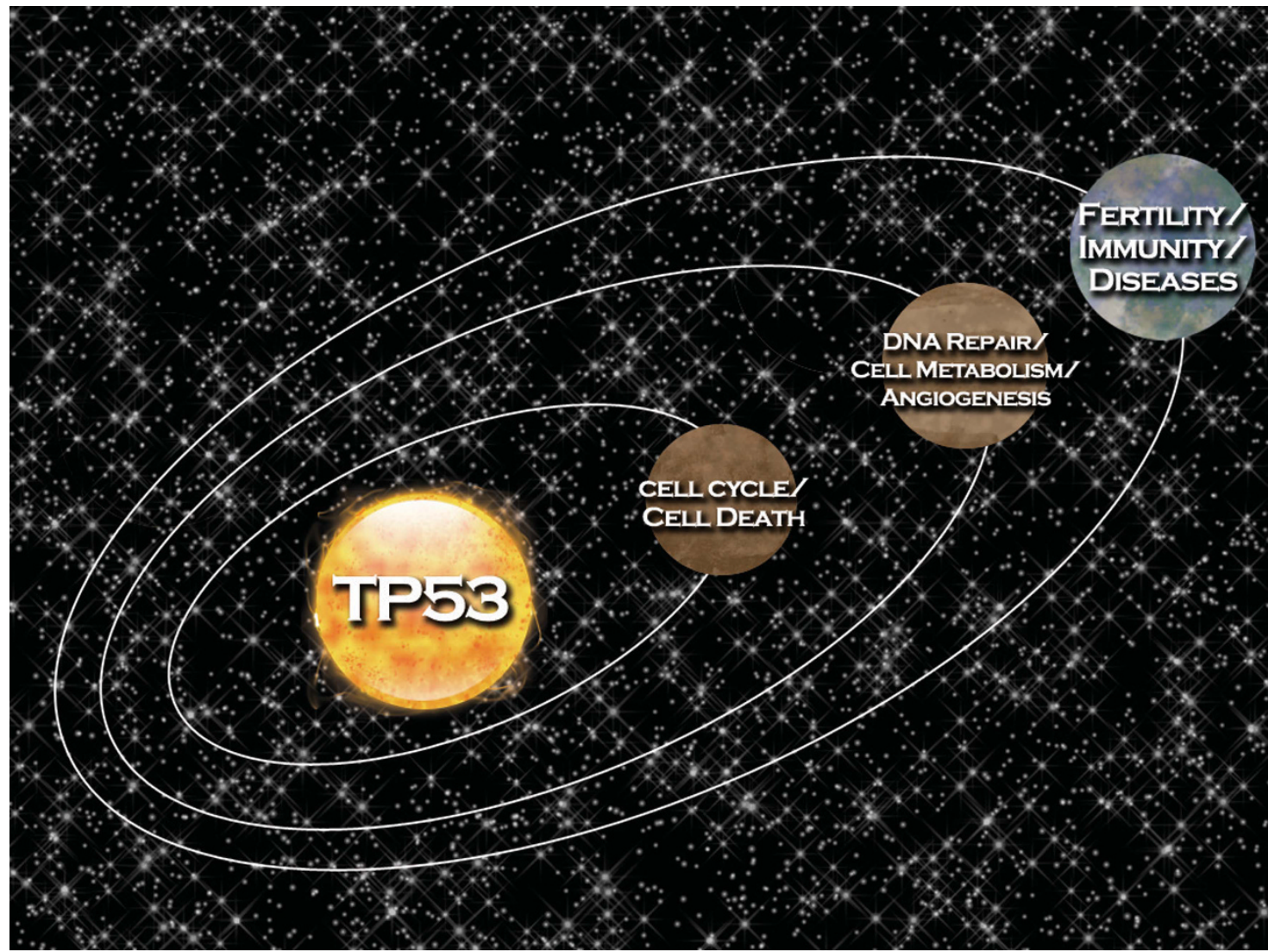

Figure 1 Expanding the universe of the p53 signaling pathway. It is well known that $p 53$ controls cell cycle, apoptosis, angiogenesis and senescence (e.g., orbits closer to the master regulator TP53). The current work by Wang et al. ${ }^{2}$ identifies not only an extensive panel of new p53 target genes involved in these processes, but also less-wellunderstood TP53 activities impacting fertility, cell motility, immunity and human diseases, such as diabetes and rheumatoid arthritis (orbits further and further away)

target genes. Importantly, 20 of the newly identified p53 target genes (e.g., STAT4) were validated using Nutlin-3 (Mdm2 inhibitor) and p53 siRNA in HCT116 $p 53^{+/+}$and p53 ${ }^{-1-}$ cells.

Network analysis placed many of these new target genes in well-established p53 pathways, including those governing cell cycle, cell survival/apoptosis and DNA damage responses, thus enriching our understanding of how p53 controls these fundamentally important processes. One such example is the identification of multiple genes involved in fertility. Earlier work by Levine and co-workers ${ }^{12}$ demonstrated that female p53-knockout mice are infertile due to a deficiency in the expression of the p53-responsive leukemia inhibitory factor gene, which encodes a cytokine that is important for implantation. Notably, some of the new p53 targets are also associated with cell signaling, metabolism and motility, as well as diverse disease states not typically ascribed to p53 function, such as rheumatoid arthritis and diabetes (see Figure 1). These findings reinforce the growing sentiment that p53 not only plays an essential role in tumor suppression but also carries out more broader functions in normal and pathological cellular processes. ${ }^{13}$ The identification of these new target genes provides an excellent foothold for moving forward in fully understanding p53 biology and its relevance to human health. Future studies will also be needed to identify novel p53 targets using different cell types and stress conditions that were not included in the present study. Lastly, physiological models for studying low-penetrant mutant p53 alleles, which may be functionally compromised rather than inactive as explored in the present study, will need to be developed.

\section{Conflict of Interest}

The author declares no conflict of interest.

Acknowledgements. I thank Emilia Pinto for intellectual discussions and Christine Zambetti for visual artwork.

1. Vousden K, Prives C. Cell 2009; 137: 413-431.

2. Wang B et al. Cell Death Differ 2014; 21: 521-532.

3. El-Deiry WS et al. Nat Genet 1992; 1: 45-49.

4. El-Deiry WS et al. Cell 1993; 75: 817-825.

5. Chen X et al. Genes Dev 1996; 10: 2438-2451.

6. Petitjean A et al. Hum Mutat 2007; 28: 622-629.

7. Liu G et al. Nat Genet 2004; 36: 63-68.

8. Ribeiro RC et al. Proc Natl Acad Sci USA 2001; 98: 9330-9335.

9. Custódio $\mathrm{G}$ et al. J Clin Oncol 2013: 31: 2619-2626.

10. Zambetti GP. J Cell Physiol 2007; 213: 370-373

11. Kato $S$ et al. Proc Natl Acad Sci USA 2003; 100: 8424-8429.

12. Hu W et al. Nature 2007; 450: 721-724.

13. Levine AJ, Hu W, Feng Z. Cell Death Differ 2006; 13: 1027-1036. 